I. UEDA

KODAI MATH. J.

6 (1983), 135-146

\title{
ON THE GROWTH OF MEROMORPHIC FUNCTIONS OF ORDER LESS THAN 1/2
}

\author{
By Hideharu UEDA
}

0. Introduction. Let $f(z)$ be meromorphic in the plane. Throughout this note, we shall assume familiarity with elementary aspects and notations of Nevanlinna's theory and in particular with the meaning of the symbols

$$
m(r, f), \quad n(r, a, f), \quad N(r, a, f), \quad T(r, f), \quad \delta(a, f) .
$$

Further we define

$$
M(r, f)=\max _{|z|=r}|f(z)|, \quad m^{*}(r, f)=\min _{|z|=r}|f(z)|,
$$

and denote by $\rho$ and $\mu$, respectively, the order and lower order of $f(z)$.

A real-valued function $L(r)$ defined for all $r \geqq r_{0}(\geqq 0)$ is said to be a slowly varying function (at $\infty$ ) if

(i) $L(r)$ is positive and continuous in $r_{0} \leqq r<\infty$

and (ii) $\lim _{r \rightarrow \infty} \frac{L(\lambda r)}{L(r)}=1$, for every fixed $\lambda>0$.

This concept was introduced by Karamata [4]. He proved that for each $\varepsilon>0$,

$$
r^{\varepsilon} L(r) \rightarrow \infty, \quad r^{-\varepsilon} L(r) \rightarrow 0 \quad(r \rightarrow \infty) .
$$

Let $f(z)$ be an entire function of order $\rho, 0<\rho<1 / 2$. If $f(z)$ is of minimal type, then a well-known theorem of Kjellberg [5] implies that

$$
\log m^{*}(r, f)>\cos \pi \rho \log M(r, f)
$$

on an unbounded sequence of $r$. If $f(z)$ is of mean type, then the following result is valid. (See [2].)

THEOREM A. Let $h(r)\left(r \geqq r_{0}\right)$ be a slowly varying function such that $h(r) \rightarrow 0$ $(r \rightarrow \infty)$ and

$$
\int_{r_{0}}^{\infty} \frac{h(t)}{t} d t=\infty
$$

If $f(z)$ is an entire function of order $\rho(0<\rho<1 / 2)$ and mean type, then

Received May 4, 1982 


$$
\log m^{*}(r, f)>\cos \pi \rho(1-h(r)) \log M(r, f)
$$

on a sequence of $r \rightarrow \infty$.

If $h(r)$ does not satisfy condition (2), then there is an entire function of order $\rho(0<\rho<1 / 2)$ and mean type for which

$$
\log m^{*}(r, f)<\cos \pi \rho(1-h(r)) \log M(r, f) \quad\left(r \geqq r_{0}\right) .
$$

In Theorem A, Barry [2, p. 45] assumes also that $h^{\prime}(r)>-O\left(r^{-1}\right)(r \rightarrow \infty)$. However, this condition is unnecessary (cf. [7]).

It is natural to consider the analogous problems to the above results for meromorphic functions. That is, for a meromorphic function $f(z)$, what can we say about the relation between $\log m^{*}(r, f)$ and $T(r, f)$ ?

In this note we shall prove the following two results.

THEOREM 1. Let $f(z)$ be meromorphic of order $\rho(0<\rho<1 / 2)$ and minimal type. Assume that there is a $\delta \in(0,1]$ such that

$$
\cos \pi \rho-1+\delta>0
$$

and

$$
N(r, \infty, f) \leqq(1-\delta) T(r, f)+O(\log r) \quad(r \rightarrow \infty)
$$

Then

$$
\log m^{*}(r, f)>\frac{\pi \rho}{\sin \pi \rho}(\cos \pi \rho-1+\delta) T(r, f)-O(\log r)
$$

on an unbounded sequence of $r$.

THEOREM 2. Let $h(r)\left(r \geqq r_{0}\right)$ be a slowly varying function satisfying $h(r) \rightarrow 0$ $(r \rightarrow \infty)$ and (2). Let $f(z)$ be meromorphic of order $\rho(0<\rho<1 / 2)$ and mean type. Assume that there is a $\delta \in(0,1]$ satisfying (3) and (4). Then

$$
\log m^{*}(r, f)>\frac{\pi \rho}{\sin \pi \rho}(\cos \pi \rho-1+\delta)(1-h(r)) T(r, f)
$$

on a sequence of $r \rightarrow \infty$.

For the case that $f(z)$ is entire-in this case we can choose $\delta=1$-Theorems 1 and 2 have been proved in [7].

\section{Lemmas}

LEMmA A. ([1, p. 189]) Let $f(z)$ be meromorphic in the plane and such that for some $\rho, 0<\rho<1$, either

$$
\pi \rho N(r, 0, f) \leqq \sin \pi \rho \log M(r, f)+\pi \rho \cos \pi \rho N(r, \infty, f)
$$


or

$$
\sin \pi \rho \log m^{*}(r, f) \leqq \pi \rho \cos \pi \rho N(r, 0, f)-\pi \rho N(r, \infty, f)
$$

for all large $r$. Then $\lim _{r \rightarrow \infty} \inf T(r, f) / r^{\rho}>0$.

LEMMA B. ([5, p. 280]) Let $f(z)$ be a nonconstant meromorphic function. Then for a given $\varepsilon>0(0<\varepsilon<1)$ and every sufficiently large value $r_{0}$,

$$
N(r, a, f) \geqq T(r, f)-2 T(r, f)^{(1+\varepsilon) / 2}
$$

for $r \geqq r_{0}$, with the possible exception of a set $E_{0}$ of values a whose capacity is at most

$$
\exp \left(-\varepsilon T\left(r_{0}, f\right)^{\varepsilon} / 4\right)
$$

LEMMA C. (cf. [7] and [2, p. 54]) Let $h(r)\left(r \geqq r_{0}\right)$ be a slowly varying function satısfyıng $h(r) \rightarrow 0(r \rightarrow \infty)$ and (2). Set $h(t)=\left(t / r_{0}\right) h\left(r_{0}\right)\left(r_{0} \geqq t \geqq 0\right)$, and let

$$
L(r)=\exp \left\{\tilde{\delta} \int_{1}^{r} \frac{h(t)}{t} d t\right\} \quad(\tilde{\delta}: \text { a positive constant }) .
$$

Consider the positıve harmonic function $H(z)$ in $\boldsymbol{C}-(-\infty, 0]$ defined by

$$
H\left(r e^{i \theta}\right)=\frac{1}{\pi} \int_{0}^{\infty} \frac{r^{1 / 2}(r+s) s^{\rho} L(s) \cos \frac{\theta}{2}}{s^{1 / 2}\left(r^{2}+s^{2}+2 s r \cos \theta\right)} d s \quad(0<\rho<1 / 2) .
$$

Then we have

$$
\begin{aligned}
& \frac{H(r)}{H(-r)}<\frac{1+[1+o(1)] \tilde{\delta} h(r) C(\rho) \cos \pi \rho}{\cos \pi \rho} \\
& \quad(r \rightarrow \infty ; C(\rho): \text { a positive constant depending only on } \rho) .
\end{aligned}
$$

If $f(z)$ is an entire function of order $\rho$, mean type and all of whose zeros are negative, then there is an unbounded sequence $r=r_{n}$ such that

$$
0<\frac{\log |f(r)|}{\log |f(-r)|} \leqq \frac{H(r)}{H(-r)}
$$

and

$$
\frac{N(r, 0, f)}{\log |f(-r)|}<\frac{1}{\pi \rho \cot \pi \rho\left\{1-\frac{\sqrt{ } 2 \pi \rho}{\tan \frac{\pi \rho}{\pi \rho}} \tilde{\delta} C_{1}(\rho) h(r)\right\}\left\{1-\frac{4 \tilde{\delta}^{2} h^{2}(r)}{\rho^{2} \tan ^{2} \pi \rho}\right\}}
$$

$\left(C_{1}(\rho)\right.$ : a positive constant depending only on $\left.\rho\right)$.

2. Proof of Theorem 1. Since $f(z)$ is of order $\rho(0<\rho<1 / 2)$ and minimal type, we deduce from Lemma A that

$$
\sin \pi \rho \log m^{*}(r, f)>\pi \rho \cos \pi \rho N(r, 0, f)-\pi \rho N(r, \infty, f)
$$


on an unbounded sequence of $r$. We denote this sequence by $\left\{r_{n}\right\}_{1}^{\infty} \uparrow \infty$.

First, we show that

$$
\log m^{*}\left(r_{n}, f\right) \geqq 0
$$

for sufficiently large $n$. By (2.1), (4) and (3)

$$
\begin{aligned}
& \sin \pi \rho \log m^{*}(r, f)+\pi \rho \cos \pi \rho m(r, 1 / f) \\
& >\pi \rho \cos \pi \rho T(r, f)-\pi \rho N(r, \infty, f)-O(1) \\
& >\pi \rho[\cos \pi \rho-1+\delta] T(r, f)-O(\log r)>0 \quad\left(r=r_{n}, n \geqq n_{0}\right) .
\end{aligned}
$$

Assume that $\log m^{*}\left(r_{k}, f\right)>0$ for some $k \geqq n_{0}$. Then

$$
m\left(r_{k}, 1 / f\right) \leqq \max _{0 \leqq \theta \leqq 2 \pi} \log \left|1 / f\left(r_{k} e^{i \theta}\right)\right|=-\log m^{*}\left(r_{k}, f\right) .
$$

From this and (2.3) it follows that

$$
\sin \pi \rho \log m^{*}\left(r_{k}, f\right)-\pi \rho \cos \pi \rho \log m^{*}\left(r_{k}, f\right)>0 .
$$

Hence $\tan \pi \rho<\pi \rho$, a contradiction. This proves (2.2), which in particular implies that

$$
m\left(r_{n}, 1 / f\right)=0 . \quad\left(n \geqq n_{0}\right) .
$$

Substituting (2.4) into (2.3), we obtain

$$
\log m *\left(r_{n}, f\right)>\frac{\pi \rho}{\sin \pi \rho}(\cos \pi \rho-1+\delta) T\left(r_{n}, f\right)-O\left(\log r_{n}\right) \quad(n \rightarrow \infty) .
$$

This completes the proof of Theorem 1 .

\section{Proof of Theorem 2; Preliminaries.}

3.1. Assume first that there is a positive number $\lambda<\rho$ such that

$$
\lim _{r \rightarrow \infty} \inf T(r, f) / r^{\lambda}=0 .
$$

Then as in the proof of Theorem 1 we deduce that

$$
\log m^{*}\left(r_{n}, f\right)>\frac{\pi \lambda}{\sin \pi \lambda}(\cos \pi \lambda-1+\delta) T\left(r_{n}, f\right)-O\left(\log r_{n}\right),
$$

where $\left\{r_{n}\right\}_{1}^{\infty}$ is a suitable increasing sequence tending to $\infty$. Since the function

$$
\frac{x}{\sin x}(\cos x-\alpha) \quad(\alpha: \text { a nonnegative constant })
$$

decreases strictly as $x \in[0, \pi / 2)$ increases, we deduce from (3.1) that 


$$
\log m^{*}\left(r_{n}, f\right)>\frac{\pi \rho}{\sin \pi \rho}(\cos \pi \rho-1+\delta) T\left(r_{n}, f\right)
$$

for sufficiently large $n$. Hence, in what follows, we may consider only the case that

$$
\lim _{r \rightarrow \infty} \inf T(r, f) / r^{\lambda}>0
$$

for all $\lambda \in(0, \rho)$.

3.2. By Lemma $\mathrm{B}$, there is a complex number $a$ such that

$$
N(r, a, f) \geqq T(r, f)-2 T(r, f)^{3 / 4} \quad\left(r \geqq R_{0}>0\right) .
$$

Put

$$
F(z)=f(z)-a
$$

It is clear that $F(z)$ satisfies all the assumptions of Theorem 2. Assume now that the conclusion of Theorem 2 holds for $F(z)$, i.e. there is a sequence $\left\{r_{n}\right\}_{1}^{\infty} \uparrow \infty$ such that

$$
\log m^{*}\left(r_{n}, F\right)>\frac{\pi \rho}{\sin \pi \rho}(\cos \pi \rho-1+\delta)\left(1-h\left(r_{n}\right)\right) T\left(r_{n}, F\right) .
$$

By (3.2), (3.4), (3.5) and (3)

$$
T\left(r_{n}, F\right), \log m^{*}\left(r_{n}, F\right)>O\left(r_{n}^{\lambda}\right) \quad(n \rightarrow \infty)
$$

for any fixed $\lambda \in(0, \rho)$. Further by (1) for each $\varepsilon>0$,

$$
r^{\varepsilon} h(r) \rightarrow \infty \quad(r \rightarrow \infty) .
$$

From (3.5), (3.6) and (3.7) follows that

$$
\begin{aligned}
\frac{\log m^{*}\left(r_{n}, f\right)}{T\left(r_{n}, f\right)} & \geqq \frac{\log m^{*}\left(r_{n}, F\right)-O(1)}{T\left(r_{n}, F\right)+O(1)} \\
& >\frac{\log m^{*}\left(r_{n}, F\right)\left[1-O\left(r_{n}^{-\lambda}\right)\right]}{T\left(r_{n}, F\right)\left[1+O\left(r_{n}^{-\lambda}\right)\right]} \\
& >\frac{\log m^{*}\left(r_{n}, F\right)\left[1-o\left(h\left(r_{n}\right)\right)\right]}{T\left(r_{n}, F\right)\left[1+o\left(h\left(r_{n}\right)\right)\right]} \\
& >\frac{\pi \rho}{\sin \pi \rho}(\cos \pi \rho-1+\delta)\left[1-(1+o(1)) h\left(r_{n}\right)\right] \quad(n \rightarrow \infty) .
\end{aligned}
$$

This implies that we may prove Theorem 2 only for $F(z)$.

3.3. Let $\left\{a_{n}\right\},\left\{b_{n}\right\}\left(a_{n}, b_{n} \neq 0\right)$ be the sequences of the zeros and poles of $F(z)$. Then we can write

$$
F(z)=c z^{p} \frac{\Pi\left(1-z / a_{n}\right)}{\Pi\left(1-z / b_{n}\right)} \equiv c z^{p} \frac{P(z)}{Q(z)} \equiv c z^{p} F_{1}(z) \quad(c \neq 0, p: \text { an integer }) .
$$


From (4) and (3.8) we deduce that

$$
N\left(r, \infty, F_{1}\right) \leqq(1-\delta) T\left(r, F_{1}\right)+O(\log r) \quad(r \rightarrow \infty) .
$$

Assume that there is a sequence $\left\{r_{n}\right\}_{1}^{\infty} \uparrow \infty$ such that

$$
\log m^{*}\left(r_{n}, F_{1}\right)>\frac{\pi \rho}{\sin \pi \rho}(\cos \pi \rho-1+\delta)\left(1-h\left(r_{n}\right)\right) T\left(r_{n}, F_{1}\right) .
$$

Then as in $\S 3.2$, we have from (3.10), (3.8), (3.6) and (3.7)

$$
\begin{aligned}
\frac{\log m^{*}\left(r_{n}, F\right)}{T\left(r_{n}, F\right)} & \geqq \frac{\log m^{*}\left(r_{n}, F_{1}\right)-O\left(\log r_{n}\right)}{T\left(r_{n}, F_{1}\right)+O\left(\log r_{n}\right)} \\
& >\frac{\log m^{*}\left(r_{n}, F_{1}\right)\left[1-O\left(r_{n}^{-\lambda} \log r_{n}\right)\right]}{T\left(r_{n}, F_{1}\right)\left[1+O\left(r_{n}^{-\lambda} \log r_{n}\right)\right]} \\
& >\frac{\log m^{*}\left(r_{n}, F_{1}\right)\left[1-o\left(h\left(r_{n}\right)\right)\right]}{T\left(r_{n}, F\right)\left[1+o\left(h\left(r_{n}\right)\right)\right]} \\
& >\frac{\pi \rho}{\sin \pi \rho}(\cos \pi \rho-1+\delta)\left[1-(1+o(1)) h\left(r_{n}\right)\right] \quad(n \rightarrow \infty) .
\end{aligned}
$$

Hence it suffices to show that the conclusion of Theorem 2 holds for $F_{1}(z)$.

\section{Proof of Theorem 2; Conclusion.}

Define $\hat{P}(z)$ and $\hat{Q}(z)$ as follows :

$$
\hat{P}(z)=\Pi\left(1+z /\left|a_{n}\right|\right), \quad \hat{Q}(z)=\Pi\left(1+z /\left|b_{n}\right|\right) .
$$

First, we show that $\hat{P}(z)$ is of order $\rho$ and mean type. By (3.8), (3.4) and (3.3)

$$
\begin{aligned}
\log M(r, \hat{P}) & \geqq m(r, \hat{P}) \geqq N(r, 0, \hat{P})=N\left(r, 0, F_{1}\right) \\
& \geqq(1-o(1)) T\left(r, F_{1}\right) \quad(r \rightarrow \infty) .
\end{aligned}
$$

On the other hand,

$$
\begin{aligned}
\log M(r, \hat{P}) & =r \int_{0}^{\infty} \frac{N(t, 0, \hat{P})}{(t+r)^{2}} d t \leqq N(r, 0, \hat{P})+r \int_{r}^{\infty} \frac{N(t, 0, \hat{P})}{t^{2}} d t \\
& \leqq T\left(r, F_{1}\right)+r \int_{r}^{\infty} \frac{T\left(t, F_{1}\right)}{t^{2}} d t=O\left(r^{\rho}\right) \quad(r \rightarrow \infty) .
\end{aligned}
$$

(4.1) and (4.2) implies that $\hat{P}(z)$ is of order $\rho$ and mean type.

Now, we apply Lemma $C$ to $\hat{P}(z)$. Then we see that there is a sequence $\left\{r_{n}\right\}_{1}^{\infty} \uparrow \infty$ such that

$$
O<\frac{\log \hat{P}\left(r_{n}\right)}{\log \left|\hat{P}\left(-r_{n}\right)\right|} \leqq \frac{H\left(r_{n}\right)}{H\left(-r_{n}\right)}<\frac{1+[1+o(1)] \tilde{\delta} h\left(r_{n}\right) C(\rho) \cos \pi \rho}{\cos \pi \rho} \quad(n \rightarrow \infty)
$$

and 


$$
\frac{N\left(r_{n}, 0, F_{1}\right)}{\log \left|\hat{P}\left(-r_{n}\right)\right|}<\frac{1}{\pi \rho \cot \pi \rho\left\{1-\frac{\sqrt{2 \pi \rho}}{\tan \pi \rho} \tilde{\delta} C_{1}(\rho) h\left(r_{n}\right)\right\}\left\{1-\frac{4 \tilde{\delta}^{2} h^{2}\left(r_{n}\right)_{\tilde{\pi}}}{\rho^{2} \tan ^{2} \pi \rho}\right\}}
$$

By (3.3), (3.4) and (3.8)

$$
(n \rightarrow \infty) \text {. }
$$

$$
N\left(r, 0, F_{1}\right) \geqq T\left(r, F_{1}\right)-T\left(r, F_{1}\right)^{3 / 4}-O(\log r) \quad(r \rightarrow \infty),
$$

and so we have

$$
\begin{aligned}
T\left(r, F_{1}\right) & \leqq N\left(r, 0, F_{1}\right)+2 T\left(r, F_{1}\right)^{3 / 4} \\
& \leqq N\left(r, 0, F_{1}\right)+O\left(r^{(3 / 4) \rho}\right) \quad(r \rightarrow \infty) .
\end{aligned}
$$

From (3.9) and (4.5) it follows that

which gives

$$
N\left(r, \infty, F_{1}\right) \leqq(1-\delta) N\left(r, 0, F_{1}\right)+O\left(r^{(3 / 4) \rho}\right) \quad(r \rightarrow \infty),
$$

$$
\log \hat{Q}(r)=r \int_{0}^{\infty} \frac{N\left(t, \infty, F_{1}\right)}{(t+r)^{2}} d t \leqq(1-\delta) \log \hat{P}(r)+O\left(r^{(3 / 4) \rho}\right) \quad(r \rightarrow \infty) .
$$

By (4.3), (4.1), (3.2) and (3.7)

$$
\begin{aligned}
\frac{r_{n}^{(3 / 4) \rho}}{\log \left|\hat{P}\left(-r_{n}\right)\right|} & \leqq O\left(\frac{r_{n}^{(3 / 4) \rho}}{\log \hat{P}\left(r_{n}\right)}\right)<O\left(\frac{r_{n}^{(3 / 4) \rho}}{T\left(r_{n}, F_{1}\right)}\right) \\
& <O\left(r_{n}^{-\varepsilon}\right) \quad(0<\varepsilon<(1 / 4) \rho) \\
& =O\left(h\left(r_{n}\right)\right) .
\end{aligned}
$$

Hence by (4.6), (4.3) and (4.7)

$$
\begin{aligned}
\log m^{*}\left(r_{n}, F_{1}\right) & \geqq \log \left|\hat{P}\left(-r_{n}\right)\right|-\log \hat{Q}(r) \\
& \geqq \log \left|\hat{P}\left(-r_{n}\right)\right|-(1-\delta) \log \hat{P}\left(r_{n}\right)-O\left(r_{n}^{(3 / 4) \rho}\right) \\
& \geqq \log \left|\hat{P}\left(-r_{n}\right)\right|\left\{1-(1-\delta) \frac{H\left(r_{n}\right)}{H\left(-r_{n}\right)}-o\left(h\left(r_{n}\right)\right)\right\} \\
& \geqq \log \left|\hat{P}\left(-r_{n}\right)\right|\left\{1-(1-\delta) \frac{1}{\cos \pi \rho}-(\tilde{\delta} C(\rho)+o(1)) h\left(r_{n}\right)\right\}
\end{aligned}
$$

On the other hand, we deduce from (4.5), (4.4) and (4.7) that

$$
(n \rightarrow \infty) \text {. }
$$

$$
\begin{aligned}
& T\left(r_{n}, F_{1}\right) \leqq N\left(r_{n}, 0, F_{1}\right)+O\left(r_{n}^{(3 / 4) \rho}\right) \\
& \leqq \log \left|\hat{P}\left(-r_{n}\right)\right|\left[\frac{1}{\pi \rho \cos \pi \rho\left\{1-\frac{\sqrt{2} \pi \rho}{\tan \pi \rho} \tilde{\delta} C_{1}(\rho) h\left(r_{n}\right)\right\}\left\{1-\frac{4 \tilde{\delta}^{2} h^{2}\left(r_{n}\right)}{\rho^{2} \tan ^{2} \pi \rho}\right\}}\right. \\
&\left.+o\left(h\left(r_{n}\right)\right)\right] \quad(n \rightarrow \infty) .
\end{aligned}
$$


Combining (4.8) and (4.9), we obtain

$$
\begin{aligned}
& \frac{\log m^{*}\left(r_{n}, F_{1}\right)}{T\left(r_{n}, F_{1}\right)} \geqq\left\{1-(1-\delta) \frac{1}{\cos \pi \rho}-(\tilde{\delta} C(\rho)+o(1)) h\left(r_{n}\right)\right\} \\
& \quad \times \pi \rho \cot \pi \rho\left\{1-\frac{\sqrt{2} \pi \rho}{\tan \pi \rho} \tilde{\delta} C_{1}(\rho) h\left(r_{n}\right)\right\}\left\{1-\frac{4 \tilde{\delta}^{2} h^{2}\left(r_{n}\right)}{\rho^{2} \tan ^{2} \pi \rho}\right\}\left\{1-o\left(h\left(r_{n}\right)\right)\right\} \\
& >\frac{\pi \rho}{\sin \pi \rho}(\cos \pi \rho-1+\delta)\left(1-h\left(r_{n}\right)\right) \quad(n \rightarrow \infty),
\end{aligned}
$$

if $\tilde{\delta}(>0)$ is sufficiently small.

This completes the proof of Theorem 2 .

5. A counterexample. It is natural to ask in Theorem 2 whether the condition (2) is necessary or not. As the answer to this question, we give the following example.

Let $h(r)\left(r \geqq r_{0}\right)$ be a slowly varying function such that $h(r) \rightarrow 0(r \rightarrow \infty)$ and

$$
\int_{r_{0}}^{\infty} \frac{h(t)}{t} d t<\infty
$$

Let $\rho$ and $\delta$ be numbers with $0<\rho<1 / 2,1-\cos \pi \rho<\delta \leqq 1$. Then there is a meromorphic function $f(z)$ satisfying the following conditions (i)-(iv).

(i) $f(z)$ is of order $\rho$ and mean type.

(ii) $\delta(\infty, f)=\delta$.

(iii) $\quad N(r, \infty, f) \leqq(1-\delta) T(r, f)+O(\log r) \quad(r \rightarrow \infty)$.

(iv) $\log m^{*}(r, f)<\frac{\pi \rho}{\sin \pi \rho}(\cos \pi \rho-1+\delta)(1-h(r)) T(r, f)$

for all sufficiently large $r$.

For convenience, we state two lemmas which will be used to construct the above example.

LEMMA D ([3]). Let $g(z)$ be meromorphic in the plane. For a measurable set $I \subset[0,2 \pi)$, define

$$
m(r, g, I)=\frac{1}{2 \pi} \int_{I} \log ^{+}\left|g\left(r e^{i \theta}\right)\right| d \theta \quad(r>0) .
$$

Then

$$
m(r, g, I) \leqq 22 T(2 r, g)|I|\left[1+\log ^{+} \frac{1}{|I|}\right],
$$

where $|I|$ is the Lebesgue measure of $I$.

LEMMA E ([2], [7]). Let $g$ be an entire function of genus zero, all of whose zeros are negatwe and such that $g(0)=1$ and $n(r, 0, g)=\left[r^{\rho} L(r)\right]$, where $L(r)$ is 
defined as in Lemma $C$. Then we have

$$
\begin{array}{r}
N(r, 0, g)=\frac{r^{o} L(r)}{\rho}\left[1-\frac{\tilde{\delta}(1+o(1))}{\rho} h(r)\right]+O(\log r) \quad(r \rightarrow \infty), \\
\log M(r, g)=r^{\rho} L(r)\left[\frac{\pi}{\sin \pi \rho}+\tilde{\delta} C_{1}(\rho)(1+o(1)) h(r)\right]+O(\log r) \\
\left(r \rightarrow \infty, C_{1}(\rho) \equiv \sum_{n=0}^{\infty}(-1)^{n}\left\{(n+1-\rho)^{-2}-(n+\rho)^{-2}\right\}\right),
\end{array}
$$

and

$$
\begin{array}{r}
\log \left|g\left(r e^{i \theta(r)}\right)\right|=r^{\rho} L(r)\left[\frac{\pi \cos \pi \rho}{\sin \pi \rho}-\tilde{\delta} \frac{\pi^{2}}{\sin ^{2} \pi \rho}-(1+o(1)) h(r)\right]+O(\log r) \\
\left(r \rightarrow \infty, \theta(r) \equiv \pi-r^{-K} \text { with } K>1\right) .
\end{array}
$$

Construction of a counterexample. Let $\alpha$ and $\beta$ be numbers such that $\alpha \cos \pi \rho>\beta \geqq 0$. Let

$$
P(z)=\prod_{n=1}^{\infty}\left(1+\frac{z}{a_{n}}\right), \quad Q(z)=\sum_{n=1}^{\infty}\left(1-\frac{z}{b_{n}}\right) \quad\left(a_{n}, b_{n}>0\right)
$$

be canonical products satisfying $n(r, 0, P)=\left[\alpha r^{\circ} L(r)\right], n(r, 0, Q)=\left[\beta r^{\circ} L(r)\right]$, respectively. Set $\delta=(\alpha-\beta) / \alpha(>1-\cos \pi \rho)$. Then we shall show that $f(z) \equiv$ $P(z) / Q(z)$ satisfies all the conditions (i)-(iv).

Using Lemma E, we have

$$
\begin{array}{r}
\log \left|f\left(r e^{i \theta(r)}\right)\right| \geqq \log \left|P\left(r e^{i \theta(r)}\right)\right|-\log Q(-r) \\
=r^{\rho} L(r)\left[\frac{\pi(\alpha \cos \pi \rho-\beta)}{\sin \pi \rho}-\tilde{\delta}\left(\alpha \frac{\pi^{2}}{\sin ^{2} \pi \rho}+\beta C_{1}(\rho)\right) h(r)-o(h(r))\right] \\
-O(\log r)>0 \quad\left(r>R_{1}\right) .
\end{array}
$$

Hence by Lemma D

$$
\begin{aligned}
m(r, 0, f) & =\frac{1}{\pi} \int_{\theta(r)}^{\pi} \log ^{+}\left|\frac{1}{f\left(r e^{i \theta}\right)}\right| d \theta \\
& \leqq 44 T(2 r, 1 / f)(\pi-\theta(r))\left[1+\log ^{+} \frac{1}{\pi-\theta(r)}\right] \\
& =44 T(2 r, f) r^{-K}[1+K \log r] \quad\left(r>R_{1}\right) .
\end{aligned}
$$

Since $T(r, f) \leqq m(r, P)+m(r, Q) \leqq \log M(r, P)+\log M(r, Q)$, we deduce from Lemma $\mathrm{E}$ and (5.1) that

$$
T(r, f)=O\left(r^{\rho}\right) \quad(r \rightarrow \infty) .
$$

In view of (5.2) and (5.3) we have

$$
m(r, 0, f)=O\left(r^{\rho+\varepsilon-K}\right) \quad(r \rightarrow \infty)
$$


for any fixed $\varepsilon>0$.

Thus (5.4) and Lemma $\mathrm{E}$ give

$$
T(r, f)=T(r, 1 / f)=\frac{\alpha r^{\rho} L(r)}{\rho}\left[1-\frac{\tilde{\delta}(1+o(1))}{\rho} h(r)\right]+O(\log r) \quad(r \rightarrow \infty) .
$$

On the other hand,

$$
N(r, \infty, f)=N(r, 0, Q)=\frac{\beta r^{\rho} L(r)}{\rho}\left[1-\frac{\tilde{\delta}(1+o(1))}{\rho} h(r)\right]+O(\log r) \quad(r \rightarrow \infty) .
$$

Combining (5.5) and (5.6) we have

Further,

$$
\delta(\infty, f)=1-\alpha / \beta=\delta .
$$

$$
\begin{aligned}
N(r, \infty, f) & =\int_{0}^{r} \frac{\left[\beta t^{\rho} L(t)\right]}{t} d t \leqq \frac{\beta}{\alpha} \int_{0}^{r} \frac{\alpha t^{\rho} L(t)}{t} d t \\
& \leqq \frac{\beta}{\alpha}[N(r, 0, f)+\log r] \leqq(1-\delta) T(r, f)+O(\log r) \quad(r \rightarrow \infty) .
\end{aligned}
$$

It remains to show (iv). Using Lemma $\mathrm{E}$ and (5.5), we have

$$
\begin{aligned}
& \log m^{*}(r, f)=\log |P(-r)|-\log Q(-r) \\
& \leqq r^{\rho} L(r)\left[\frac{\pi(\alpha \cos \pi \rho-\beta)}{\sin \pi \rho}-\tilde{\delta}\left(\frac{\alpha \pi^{2}}{\sin ^{2} \pi \rho}+\beta C_{1}(\rho)\right) h(r)+o(h(r))\right]+O(\log r) \\
& \leqq\left[\frac{\pi(\alpha \cos \pi \rho-\beta)}{\sin \pi \rho}-\tilde{\delta}\left(\frac{\alpha \pi^{2}}{\sin ^{2} \pi \rho}+\beta C_{1}(\rho)\right) h(r)+o(h(r))\right] \\
& \quad \times \frac{\rho(T(r, f)-O(\log r))}{\alpha\left[1-\frac{\tilde{\delta}}{\rho} h(r)-o(h(r))\right]}+O(\log r) \\
& =\frac{\rho}{\alpha}\left[\frac{\pi(\alpha \cos \pi \rho-\beta)}{\sin \pi \rho}-\tilde{\delta}\left(\frac{\alpha \pi^{2}}{\sin ^{2} \pi \rho}+\beta C_{1}(\rho)-\frac{\pi(\alpha \cos \pi \rho-\beta)}{\rho \sin \pi \rho}\right) h(r)\right. \\
& +o(h(r))] T(r, f)+O(\log r) .
\end{aligned}
$$

Here, we note that

$$
A(\rho) \equiv \frac{\alpha \pi^{2}}{\sin ^{2} \pi \rho}+\beta C_{1}(\rho)-\frac{\pi(\alpha \cos \pi \rho-\beta)}{\rho \sin \pi \rho}>0 .
$$

In fact,

$$
\begin{aligned}
A(\rho) & =\frac{\pi^{2}}{\sin ^{2} \pi \rho}\left\{\alpha+\beta \frac{\sin ^{2} \pi \rho}{\pi^{2}} C_{1}(\rho)-\frac{\sin \pi \rho(\alpha \cos \pi \rho-\beta)}{\pi \rho}\right\} \\
& =\frac{\pi^{2}}{\sin ^{2} \pi \rho}\left\{\alpha\left(1-\frac{\sin 2 \pi \rho}{2 \pi \rho}\right)+\beta\left(\frac{\sin \pi \rho}{\pi \rho}+\frac{\sin ^{2} \pi \rho}{\pi^{2}} C_{1}(\rho)\right)\right\}
\end{aligned}
$$




$$
\begin{aligned}
& =\frac{\pi^{2}}{\sin ^{2} \pi \rho}\left\{\alpha\left(1-\frac{\sin 2 \pi \rho}{2 \pi \rho}\right)+\beta \frac{\sin ^{2} \pi \rho}{\pi^{2} \rho^{2}}\left(\frac{\pi \rho}{\sin \pi \rho}\right.\right. \\
& \left.\left.\quad+\rho^{2} \sum_{n=0}^{\infty}(-n)^{n}\left[(n+1-\rho)^{-2}-(n+\rho)^{-2}\right]\right)\right\} \\
& \geqq \frac{\pi^{2} \alpha}{\sin ^{2} \pi \rho}\left(1-\frac{\sin 2 \pi \rho}{2 \pi \rho}\right)+\frac{\beta}{\rho^{2}}\left[\frac{\pi \rho}{\sin \pi \rho}+\rho^{2}\left(\frac{1}{(1-\rho)^{2}}-\frac{1}{\rho^{2}}\right)\right] \\
& =\frac{\pi^{2} \alpha}{\sin ^{2} \pi \rho}\left(1-\frac{\sin 2 \pi \rho}{2 \pi \rho}\right)+\frac{\beta}{\rho}\left(\frac{\rho^{2}}{(1-\rho)^{2}}+\frac{\pi \rho}{\sin \pi \rho}-1\right)>0 .
\end{aligned}
$$

Since $T(r, f) \sim(\alpha / \rho) r^{\rho} L(r)$,

$$
\log r=o(h(r)) T(r, f) \quad(r \rightarrow \infty) .
$$

Therefore, choosing $\tilde{\delta}$ large enough, we obtain from (5.7), (5.8) and (5.9)

$$
\log m^{*}(r, f) \leqq \frac{\pi \rho}{\sin \pi \rho}(\cos \pi \rho-1+\delta)(1-h(r)) T(r, f) \quad\left(r \geqq R_{2}\right) .
$$

Remark. The method of this section can be used also when we prove the following result.

Let $h(r)$ be given as in Theorem 2. Let $\rho$ and $\delta$ be numbers with $0<\rho<1 / 2$, $1-\cos \pi \rho<\delta \leqq 1$. Then there is a meromorphic function $f(z)$ satisfyng the following (i)-(iv).

(i) $f(z)$ is of order $\rho$ and minimal (maximal) type.

(ii) $\delta(\infty, f)=\delta$.

(iii) $N(r, \infty, f) \leqq(1-\delta) T(r, f)+O(\log r) \quad(r \rightarrow \infty)$.

(iv) $\log m^{*}(r, f) \leqq \frac{\pi \rho}{\sin \pi \rho}(\cos \pi \rho-1+\delta)(1+h(r)(-h(r))) T(r, f)$

for all sufficiently large $r$.

\section{REFERENCES}

[1] Anderson, J. M., Regularity criteria for integral and meromorphic functions, J. Analyse Math. 14 (1965), 185-200.

[2] BARRY, P.D., On the growth of entire functions, Mathematical essays dedicated to A. J. Macintyre, Ohio Univ. Press (1970).

[3] EDREI, A. AND FUCHS, W.H.J., Bounds for the number of deficient values of certain classes of functions, Proc. London Math. Soc. 12 (1962), 315-344.

[4] Karamata, J., Sur un mode de croissance régulière des fonctions, Mathematica (Cluj) 4 (1930), 38-53.

[5] KJellberg, B., A theorem on the minimum modulus of entire functions, Math. Scand. 12 (1963), 5-11.

[6] Nevanlinna, R., Eindeutige Analytische Funktionen, second edition, Berlin (1953). 
[7] Ueda, H., On the growth of entire functions of order less than 1/2, Kodai Math. J. vol. 5 no. 3 (1982), 370-384.

Department of Mathematics Daido Institute of Technology

Daido-cho, Minami-Ku, Nagoya, Japan 